\title{
Data Stream Mining Between Classical and Modern Applications: A Review
}

\author{
Ammar Thaher Yaseen Al Abd Alazeez \\ Computer Science Department, College of Computer Science and Mathematics, University of Mosul, Mosul, \\ IRAQ \\ E-mail: ammarthaher@uomosul.edu.iq
}

(Received May 07, 2021; Accepted June 31, 2021; Available online December 01, 2021)

DOI: 10.33899/edusj.2021.130093.1158, (c) 2021, College of Education for Pure Science, University of Mosul.

This is an open access article under the CC BY 4.0 license (http://creativecommons.org/licenses/by/4.0/).

\begin{abstract}
Data mining (DM) is an amazing developing with incredible chances to advantage institutions centre of main data of information accumulated of conduct their customer and expected customer. DM identified data included in information which questions and summaries cannot viably discover. DM is a straight way to examining data of periodic data records and summing up in useful information information could be used in expand outputs, reduction costs, or both. DM allows clients to verify data of various measurements or points, classify it, and sum up the connections recognized. There are four types of DM: 1) Classification and regression, 2) Clustering, 3) Association Rule Mining, and 4) Outlier/Anomaly Detection. Tending to the velocity part of Big Data (BD) has as of late pulled in a lot of revenue in the investigation local area because of its critical effect on information from pretty much every area of life like medical services, financial exchange, and interpersonal organizations, and so on. A lot of paper works verified the velocity challenge via stream mining data. The majority of streaming mining data articles centres around adjusting primary classifications of algorithms, methods and techniques of classic information to the modified information circumstance. This research explores widely the latest literature of mining stream data field recognizes the essential ready nodes supporting variance founded methods. This study not simply benefits examiner to make strong assessment subjects and separate gaps in the field yet moreover helps specialists for DM and BD application structure headway.
\end{abstract}

Keywords: data stream mining; mining algorithms; big data

$$
\begin{aligned}
& \text { تتقيب البيانات المتدفقة بين التطبيقات القديمة والحديثة: مقال مراجعة } \\
& \text { عمار ظاهر ياسبن طه عبد العزيز } \\
& \text { قسم علوم الحاسوب، كلية علوم الحاسوب و الرياضيات، جامعة الموصل، الموصل، العراق }
\end{aligned}
$$

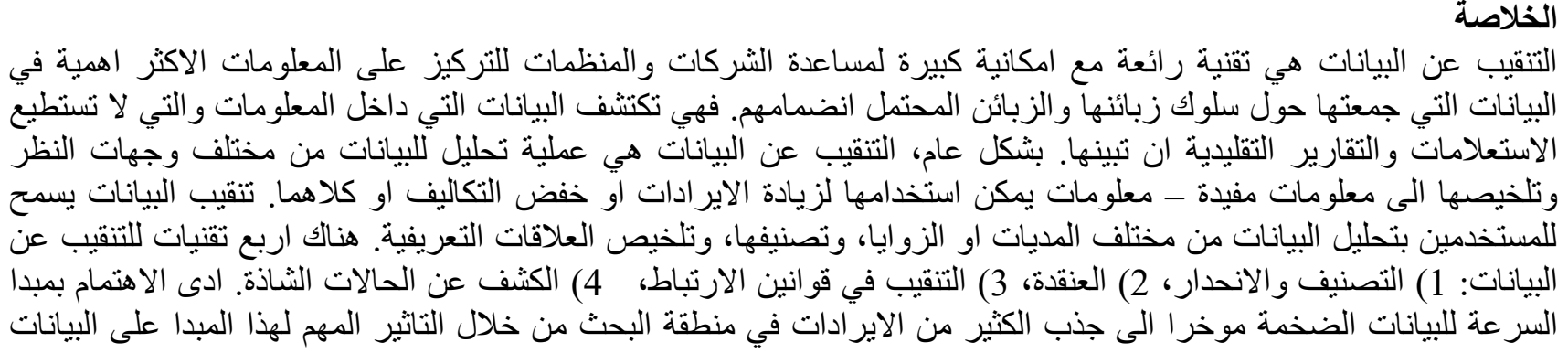




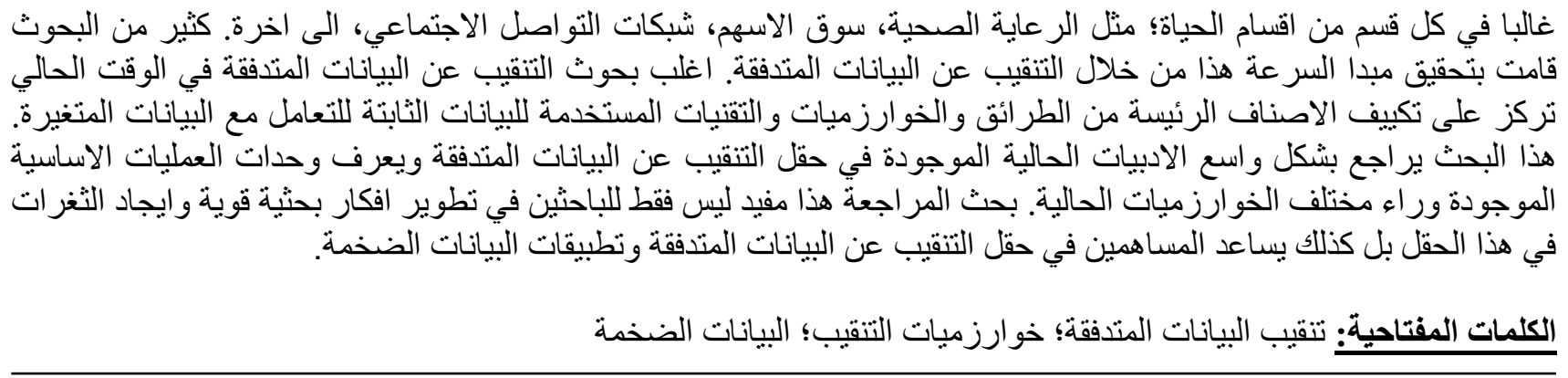

\section{Introduction}

Data stream mining has as of late a tremendous measure of consideration. It is intended to handle velocity principle in Big Data. Streaming data is useful fixing attain information that forces solitary pass limitation when irregular admittance into information isn't possible (one pass). The pace of data arriving, only the pace of data controlling, might change of one system to another. For specific system, the arrive and preparing in information might developed in separate batch investigation style, others demand ceaseless simonise examinations; now, again it demand quick action over handling in demanding streaming data, for instance, automatic managing farms. Mining data streams might describe the road of discovering covered up architecture in an enormous size of unlimited streaming data. This state demand mining algorithms should verify acknowledge reality imperatives should make the selection of finding secret patterns in concentrates. Data arriving in streams regularly contains outliers. In this case, it develop data stream algorithms to distinguish the outliers only as patterns [1].

Data streams are described by steadily expanding volumes, consistently advancing qualities and capricious rates of appearance. Stock exchange costs are continually going up and down. Clients' shopping practices change throughout the long term. The fundamental subjects of conversation on Twitter can change starting with one hour then onto the next. This fluid condition of information forces extra requests on old style mining strategies to rapidly measure and sum up the immense measure of persistently evolving information. It additionally requires a capacity to adjust to changes in the information dispersion, recognizing arising designs or erasing obsolete ones, and distinguishing oddities in the information. Such requests make data stream mining a significant and current issue to tackle. In view of the preparing time requirement, numerous conventional mining methods can't be straightforwardly applied and subsequently must be altered to work adequately and productively in a dynamic information climate [2][3]. Consistently pressing requirement of growing add methods of streaming data mining which could manage elements in continually reaching information and expand to quantity information focuses, in actuality, systems.

This research is proposed to accomplish the accompanying explicit targets: To explore and comprehend computational issues about Big Data investigation when all is said in done and data stream mining specifically (velocity of Big Data). To accomplish the point and goals of the exploration, this research will be directed through the accompanying strategies: Investigating the present status of-the-art of the writing by leading a broad and orderly audit of the applications of DM, Big Data, and especially in streaming data mining.

\section{Streaming Data and Big Data}

\subsection{Prosperities of Big Data}

Big data might be depicted using 3 primary attributes named "3Vs", for example volume, variety, and velocity. Volume alludes information quantity. Aftereffect in PC computerization of day by day information preparing exercises and the utilization of information move stages like the Internet, always expanding measures of information are gathered and put away. Information is made from different sources including on the web exchanges, sensors, person to person communication exercises, wellbeing records, enumeration, logical examinations and examinations, live streaming 
media materials, and so forth. For instance, moment, assessed that more than 99 add records of LinkedIn, more than 500,000 Tweets in Twitter created, more than 2,000,000 bits substance sent Facebook, and more than 70 hours new recordings transferred YouTube. Only 2003, five Exabyte information have been made; these days measure information might produced only 1 or 2 days. Variety alludes distinctions of information configurations portrayals. Customarily, information regarding numbers and character strings is caught and put away as organized records of fixed length in social tables and documents. These days, information can be of different intricacies, for example, site web recorded XML (semi-structured), sound, video, and manuscript reports (unstructured). Different organizations from information present difficulties of addressing, putting away and recovering such information effectively and proficiently. Velocity alludes to the changing idea of the information which incorporates changing the speed of information coming, changing the worth of information, and changing the examination speed. Information can be dynamic as in new information are collected, and existing information are erased from information records at various rates. Continuous frameworks, for example, tactile organization and securities exchange produce huge volumes of on-going stream information, representing another test to examine information of this sort [4][5].

Our research will focus on the velocity properties of Big Data (data stream) and especially accentuation on the changing the worth of information.

\subsection{Big Data: Promises and Challenges}

Every characteristic of Big Data (3V's) presents serious specialized difficulties for big data analysis. Despite the fact that data mining and AI are the correct headings for large information investigation, the sheer sizes of huge datasets, for example enormous information volume, make many existing methods insufficient to increase. The methods quite often need to depend on the computational force of the machine equipment to finish the complex logical errands inside an adequate timeframe. The variety of Big Data designs implies which a lot of information mining methods which think about internes information of simple database records in lines and columns in structured information. Absence of information quality for genuine enormous information can likewise make challenges for significant investigation. The modified data in/out of Big Data, in other words, Big Data velocity, additionally implies which a lot of existing information mining methods which work with information in a classic assortment won't increase for the steady changes at various velocities [4][6][7].

Because of the sheer size of the difficulties, this research can just address a portion of the specialized difficulties identifying with the velocity part of the Big Data qualities.

\section{Data Mining (DM)}

Data mining is an interaction of finding covered up similarities relationship covered assortments of information which helpful in expected systems. Generally, three significant assignments of mining data: clustering, association rule mining, and classification. Classification way toward allotting new information data point in bunch in existing class marks. Process includes initially constructing a model for order, for example, a decision tree by utilizing a bunch of named information models, and afterward foreseeing the name of a concealed information record by utilizing the model. Association rule mining finds assuming/proclamations that portray critical acquainted connections among include values that happen more often than other potential affiliations. Clustering is to discover significant objects among comparative information focuses where the degree of similitude among the individuals from a similar gathering is higher than the likeness among the individuals from the various gatherings. Association rule mining and Clustering are accepted as solo learning disclosure in comparable gatherings of solid affiliations which unflawed of existing class difference. Then again, arrangement is viewed as managed realizing while order prototype is developed anticipate class result in existing information data point [8][9][10]. 


\section{Mining Stream Data: Issues and Promises}

Mining stream data is energized in arising applications including enormous datasets. Coming up next are a portion of these applications [11]:

1. Financial exchange analysis: Prices of stocks are expanding and diminishing over the long haul. Value information persistently created continuously during exchanging. Yet, some stock costs flood arrive simultaneously of certain time-frames. An example, stocks might gathered utilizing stream mining methods. Data would useful in financial business coordinate their stock portfolios and choose which legitimate time in selling of purchasing.

2. Network Sensors: A famous network sensors are medical care framework. An instance, modernized emergency clinics outfitted in patient reconnaissance framework to enhance medical care services profitability. Because of sensor hardware simply save adjusted information the natural state can't distinguish signs, framework ought to dissect medical services data streams in an on-going and concentrate helpful data for remedial experts to recognize significant occasions.

3. Water distribution networks: The assessment of the drinking water-quality is in a perfect world extraordinary scale and a constant reconnaissance system. Water-purity proportion in water's properties alludes into real, organic, substance highlights. Water-purity estimations introduce gigantic measure stream information which should handle. Li et al carried out explores different avenues regarding two diverse dissemination organizations. The main organization is a genuine water circulation framework with 129 hubs. The subsequent organization incorporates 920 stations, shows up at the middle for water frameworks at the University of Exeter. The creators introduced a method that ceaselessly extricated delegates out of gigantic data streams. To persistently distinguish the delegates in a productive manner, the creators applied online agent change measures just when significant development happens.

Data streams have fundamental highlights, like boundless size, consecutive request, and dynamical updates. Accordingly, creating compelling data stream methods is basic for the investigation of such information. Simultaneously, the entirety in characteristics of streaming data in addition to realities which streaming data are non-deterministic consistently include anomalies and commotions represent a critical genuine specialized test to mining streaming data.

\subsection{Data Stream (DS) Definition}

A data stream is inexactly considered as a constant succession of requested information produced as identified occasions continuously. Instead of static information, the data stream is dynamic as in new information focuses continually reach, obsolete information data points are continually deleted of the whole data. All the officially, an stream data $S D$ is addressed as a n-dimensional limitless rundown of data points $S_{1}, S_{2}, \ldots, S_{n}, \ldots$, produced at an once, $t_{1}, t_{2}, \ldots, t_{n}, \ldots$ separately, i.e.:

$$
\begin{aligned}
D S=<S_{1}, t_{1}> & ,<S_{2}, t_{2}>, \ldots,<S_{n}, t_{n}>, \ldots \\
= & \left(s_{11}, s_{12}, \ldots, s_{1 d}, t_{1}\right),\left(s_{21}, s_{22}, \ldots, s_{2 d}, t_{2}\right), \ldots,\left(s_{n 1}, s_{n 2}, \ldots, s_{n d}, t_{n}\right), \ldots
\end{aligned}
$$

Instances of data streams incorporate streamed media information like online news, video clips, site pages, phone records, sensor network information, and monetary exchanges [12][13].

\subsection{Data Streams (DS) Characteristics}

In light of the extremely powerful nature of streaming data, it is impossible to aggregate remake entire prototype every time period in ground-breaking visions which obtained of some data gets useless. Subsequently, learning methods of streaming data should gradual in demand. A particularly steady learning method should have the option to update as inverse in re-form exist prototype with address lately reached data vision only useless data. To feature the augmentation property of data streams, there exists a current model $C C=\left\{c_{1}, c_{2}, \ldots, c_{m}\right\}$ made by an data stream method A from DS. Considering a recently shown up information lump $D=\left\{d_{1}, d_{2}, \ldots, d_{t}\right\}$, the method An updates the current model CC into another model $C C^{\prime}=\left\{c^{\prime}{ }_{1}, c^{\prime}{ }_{2}, \ldots, c^{\prime}{ }_{p}\right\}$ by either relegating a portion of the 
recently shown up information objects in $\mathrm{D}$ to a portion of the current model $\left\{c_{1}, c_{2}, \ldots, c_{m}\right\}$ or to bunch some recently arrived information objects into another model $c^{\prime}{ }_{j}[11]$.

The principle attributes of the data streams involve:

- New information focuses arrive constantly at various paces.

- Existing information focuses may go downhill and might be taken out prepared.

- The volume of streaming data enormous most likely infeasible.

- The information age interaction might not acknowledge non-fixed, for example likelihood appropriation may change over the long haul.

- Despite the fact that there is a period grouping of the data streams, there is no power over the arrangement wherein the information focuses should be prepared inside a similar piece of information.

The dynamic idea of data streams may demonstrate the accompanying:

- The hidden patterns behind static information continue as before while those behind dynamic information do change. This implies the patterns in the static information can be found through disconnected preparing, while designs found with a more established variant of the powerful information should be refreshed considering the new information change, for example an Internet processing.

- It is too exorbitant to even think about discarding existing patterns mined from the information before and re-find the new patterns, especially when the speed of information change is quick. Along these lines, answers for dynamic information mining should be gradual as in the current patterns are adjusted productively to oblige the progressions to the information.

\subsection{Data Stream (DS): General Processes Framework}

Nguyen et al [11] introduced a conventional model for data streams mining (see Figure 1(adopted from [11])). At the point when data streams come (stage 1), a buffer is utilized to save the appearance information focuses (stage 2). To keep up the yields considering recently arrived information in the buffer, the framework may apply one of an alternate time window model (landmark, shifted-timed, blurred, and sliding-window models, they will clarify later) (stage 3). At that point, the stream handling engine (stage 4) will apply a mining method (stage 5) to make yields utilizing any processing methodologies (incremental or two-stage learning, clarify in following) (stage six) and include the rundown in yields of an information saved memory (stage seven). Distinctive structure of data, for example, prototype array, cluster feature vector, centre tree, and grid data utilized previously (they will clarify later). From that point forward, when careful rules are met, for instance, a client's solicitation or after a particular period; the framework will handle the rundown and yield surmised results and carry out stream-approval ways to deal with assess the nature of coming about yields (stage 8). In the long run, we will get the last yields (stage 9). 


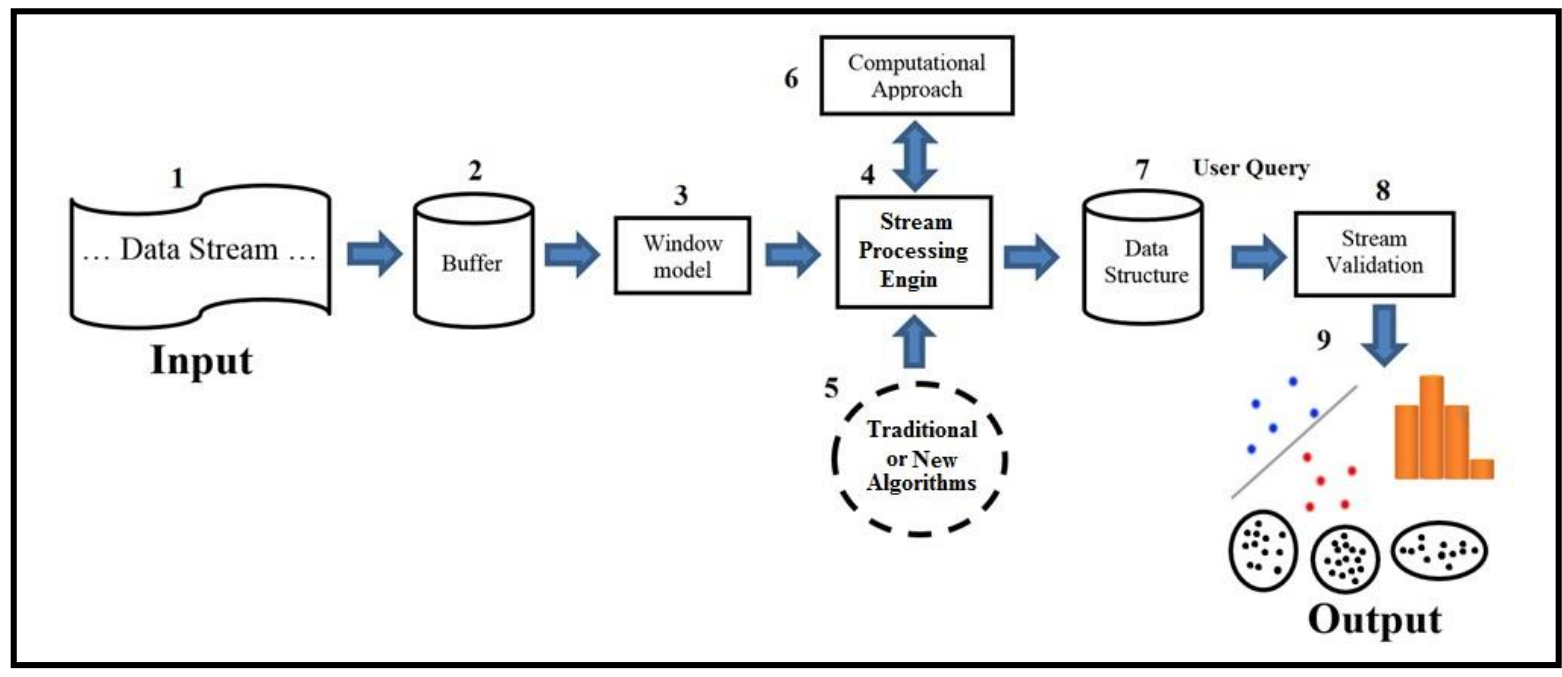

Figure 1: Typical prototype mining streaming data (modified from [11]))

\subsubsection{Streaming Data Algorithms and Window Prototypes}

A lot of streaming data draws near, later information of stream might appear in presence of add bearings or modifying information conveyance. Systems that apply comparable importance terminated add information don't get the advancement of streaming data. Streaming data are conceivably limitless, it is possible simply ready deal with small portion of the entire data streams [11]:

\section{Landmark Window Model}

The landmark window model is keen overall of the data streams from the start time-unit 1 to the current time-unit $c t$. Handling a stream dependent on milestone windows requires keeping up disconnected squares (pieces) of the data streams. landmark might be distinguished either regarding the time span (for instance, hourly, day by day or week by week) or as far as the quantity of perceptions since the last landmark.

\section{Shifted-Time Window Model}

The shifted time window model is some other time window model used to keep up yields in the data stream methods. It executes variation levels of granularity regarding the new information, for example $c t$ is a current time, $c t-1$ is time moving one time-unit to the past, $c t-2$ is time moving double cross units to the past, etc. Shifted time window generally saves the entire dataset and presents a decent compromise between store capability and productivity.

\section{Blur Window Model}

Blur window model considers the latest data by relating loads to information focuses from the data streams. In this model, every information point puts on an alternate weight adjusting to its coming time so late activities get bigger loads than past ones. This model is diminishing the impact of lapsed activities on the mining yields.

\section{Slide-Window Model}

The sliding window model just stores the new information focuses from stream information in the memory, and other information focuses are taken out. The mining results are subject to the size of the window (the size of the window can be dynamic or fixed). 


\subsubsection{Streaming Data Algorithms and Computational Methods}

Methodologies for data stream mining include [11]:

\section{Gradual Learning Approach}

The mining model in this methodology gradually develops to fit changes in the approaching information. As such, at a specific time after the fresh introduction information is gotten, the mining methods keep a refined model. The yields are consistently fit to be gotten back to the client. For example, Guha et al assessed a piece of arriving information focuses to change the current model by refreshing the heaviness of each yield with another one.

\section{Two-stage Learning Approach}

The essential thought of two-stage learning is to part the mining interaction into two stages. In the principal stage, rundowns of information focuses, known as pseudo model, are refreshed and kept up in the memory in an on-going sense. In the subsequent stage, the mining cycle works on the put away outlines at whatever point a client asks an inquiry. For instance, Aggarwal et al proposed an onlinedisconnected technique called CluStream. Its online stage sums up the factual data about the data streams progressively. The offline stage utilizes the outline insights to perform at whatever point mentioned.

\subsubsection{Data Abstraction and Data Structure of Data Streams Algorithms}

Data abstraction, generally, implies gathering information protests together then utilizing one item to address a gathering of them. Segment abstraction deliberation intends to put a gathering of sections together and utilize one segment to address those segments. This thought was utilized in PCA (principal component analysis) by utilizing fundamental element credits to gathering and produce a meta property. Information reflection moves somewhat away from information level to a more significant level, for example more dynamic and easier with less subtlety than the actual information. As a result of a tremendous measure of information transition in data stream applications, the data stream methods will in general utilize information deliberation (called data structure) to sum up information, for example metadata. It incorporates four sorts model array, feature vector, centre set tree, and data grid [14][15]:

\section{Model Array}

Model exhibit is a straightforward summarisation structure and utilized in a few data stream methods, for example, STREAM and Stream-LSearch methods. It is a variety of items that sum up the information parcelling, for example the quantity of yields, the quantity of information focuses, and the centroid. This model is utilized for additional investigation.

\section{Feature Vector}

The feature vector is another information summary used to sum up a lot of information focuses in data stream methods. The primary distinguishing proof of an element vector was in the BIRCH method and called a cluster feature (CF) vector. This vector has three components: (N,LS,LSS), where $N$ is the quantity of information focuses, $L S$ is the direct amount of the information focuses, and LSS is the amount of squared information focuses. These three components permit the ascertaining measurements to be utilized for additional investigation.

\section{Centre-Set Tree}

A core/centre set tree is a twofold tree applied in StreamKM++ method used to sum up information objects of yields. By utilizing a centre set tree each tree node $i$ incorporate the accompanying components: $\left(E_{i}, X^{p i}, N_{i}, S S E_{i}\right)$, where $E_{i}$ is a group of information focuses; $X^{p i}$ is a model of $E_{i} ; N_{i}$ is the quantity of information focuses in $E_{i}$; and $S S E_{i}$ amount squared far information focuses $E_{i}$ of $X^{p i}$. $E_{i}$ just preserved in node hubs of centre tree, because information data record internal hub distinguished concatenate information data record youngster hubs. A centre set tree vector is utilized for more investigation.

\section{Data Grid}


Every information data record $\mathrm{x}$ in $\mathrm{T}$ identified with a thickness boundary which decreases up the long haul, $D\left(x^{j}, T\right)=\lambda^{T-T^{j}}$, where $\lambda \in(0,1)$ is a rot boundary. The power of the cell $\mathrm{c}$ at time $D(c, T)$, is acquired by the expansion of the adjusted densities of each information point that is planned to c previously or at time time $T(E(c, T))$, as portrayed in $D(c, T)=$ $\sum_{x \in E(c, T)} D(x, T)$. Every lattice cell is summed up by a vector $\left(T_{g}, T_{m}, D\right.$, lable, status $)$, where $T_{g}$ new time altered, $T_{m}$ new time erased, $\mathrm{R}$ cell force new adjusted, data record class-name, status shows framework typical irregular. Information lattice group utilized in additional investigation.

\section{Merging Strategies for Overlapped Outputs in Data Streams Algorithms}

Consolidating covered models that may arise during the refreshing stage is quite possibly the main strides in data stream mining. It affects the accuracy of the subsequent model. There exist three principle techniques of combining: coordinating, ascertaining the contingent likelihood of convergence zones, and utilizing radii. Spiliopoulou et al introduced a procedure that processes the coordinating between yields to catch their development. Yields are possibly combined in the event that they share at any rate a portion of their participations. Oliveira and Gama rather ascertain the restrictive likelihood for each pair of yields acquired at the sequential time and contrasts that and a predefined blending edge of 0.5 . The BIRCH method concludes whether to add another information point into the past yields relying upon the span characterized as the normal separation from individuals to the centroid. The DenStream method likewise utilized a comparable procedure dependent on the range which for this situation addresses the normal separation from the information focuses inside a miniature model to the centroid [16].

\section{Anomalies Detection in Data Stream Algorithms}

There were explicit endeavours to distinguish the anomaly protests in the data streams. Thakran and Toshniwal introduced a method that joined the K-Means standards DBSCAN standards decide exception. Method utilised as well as weighted K-Means method in weighting credits deciding anomalies. Koupaie et al created two answers for distinguishing anomalies in data streams. The principal arrangement misuses both grouping and characterization procedures: the information focuses are first assembled into groups utilizing the K-Means technique. Anomalies that are a long way from the centroids (contingent upon a limit) are likewise recognized. At that point both group individuals and outliers are marked. From that point forward, a SVM grouping model is worked to characterize anomalies. The subsequent arrangement applies a grouping method with two equal stages. The principal online stage execute K-Means algorithm grouping information to exist piece. In this gathers, little forms and information focuses distance of others are accepted anomalies put away in additional utilization subsequent stage. The $2^{\text {nd }}$ disconnected stage joins recently identified anomalies of anomalies of exist window piece and demand last anomalies. Kontaki et al presented a method called AMCOD identify anomalies in streaming data. Sliding-window technique focus of far-based anomalies. Thought of this method article $\mathrm{x}$ is accepted as an anomalies not as much as $\mathrm{n}$ closer things presented a good ways off $\mathrm{R}$ of $\mathrm{x}$. In any case, correcting the 2 edges $(\mathrm{n}, \mathrm{R})$ difficulties of method. Additionally, a ton of applicant anomalies that should save concluding genuine anomalies. New method called SAIC was presented of Zheng et al to grouping self-assertive figures automatic dataset groups. Incorporates learning after-preparing stages. Learning stage constantly distinguishes the competitor groups after-preparing stage eliminates the anomalies by using accumulate worth group quantity cycles. At end of the day, eliminates the limit focuses relying upon an edge [17][18]. 


\section{Concept Drift in Data Streams Algorithms}

Concept drift implies an balancing of the history of data changing. In streaming data mining, concept drift consider a natural change of data. The balancing of the prototype, an instance, first or a concatenation of conveyances, presenting the examples of every stream (and frequently happen) updated after time period. Naming, concept drift resulted certainty which the design incrementally with previous data figure $(t)$, which do not, at current time legitimate in reflected under perception of time period $(t+1)$ neither $(t+2)$ which might address new connection of nearness among information in streaming data [19][20].

\section{Discovering Persistency in Streaming Data}

Relying on methodologies developed, if gradual learning, two-stage learning, founding methods of streaming data if produce latest perspective on yields or produce a view at a client inquiry point. Be that as it may, there is a little endeavour in the two ways to deal with keep a memorable path of the yield models over the long haul past the outcomes up to the current information piece. In the event that a particularly noteworthy path is kept up, the steadiness of certain can be investigated by mining the yields gathered throughout a grouping of time focuses. Solidness of yield in static datasets has been well-informed and perceived [21]. Additionally, distinguishing persistency in data streams has been appropriately concentrated in. There are numerous potential applications that can profit by discovering constant in data streams. Online media occasion following like birthday events, objects following like vehicles and rockets from video recordings, security observing utilizing CCTV cameras in distinguishing unusual articles like unattended packs against a steady foundation, and patient circumstance checking in clinics are a couple of numerous potential models [22].

The major contrast of two-stage learning, second-request learning of streaming data might be explained. Two-stage learning methods attempt in find last in numerous model (miniature). It is accordingly still a first-request gaining from information to yields. The second-request learning methods, then again, mean to discover tenacious that exist through a succession of continuous outcomes. At the end of the day, it takes as information sources a succession results and recognizes as yields that endure throughout the entire time-frame [23][15].

\section{Necessities of Data Stream Mining Algorithms}

It is hard for the exploration local area to concede to a group of necessities for data stream methods which prerequisites might utilized in benchmark of assess viability in new methods. In any case, a few attractive prerequisites seem to have been agreed [24] which will likewise be carefully thought to be in this exploration:

- Iteratively refreshing outcomes. The subsequent ought to be constantly and over and over refreshed to oblige changes brought about by the recently arrived information.

- Building and refreshing models productively. The transient idea of data streams proposes that information arrive at a high speed and mining task should be finished inside a severe time limit to synchronize with the information changes.

- Having the option to deal with advancements and the concept drift considering the fresh introductions and obsolete information objects.

- Making the model accessible whenever, either utilizing the steady methodology or the twostage learning approach.

- Distinguishing the presence of anomalies. This specific necessity might be adequate yet not fundamental.

- Giving a minimized model portrayal which develops all the more gradually with the quantity of information focuses prepared.

\section{Data Stream Mining Algorithms}

Data stream methods were initially begun over twenty years prior. Many examination papers have been distributed in the writing highlighting in excess of 100 data stream methods. An outline table of the methods surveyed is given in Table 1. In addition, many overview papers, for example, [25], [26], [27], [11], and [28], tending to different parts of the field, have been distributed. 
Research work in this field has been led under various names. This writing survey incorporates the exploration work in data stream, dynamic information, real time information, and steady information. Although every one of these terms share a similar thought, each term mirrors the accentuation of the work in a specific part of variable information. Data stream is identified with the idea of the information, for example persistently arriving information focuses, though continuous and dynamic information mirror the idea of the proposed application. The steady information is one of the computational methodologies of data stream. The goal is limiting the examining and computing exertion of recently added information into the data warhorse [29]. 
Journal of Education and Science (ISSN 1812-125X), Vol: 30, No: 5, $2021(30-43)$

\begin{tabular}{|c|c|c|c|c|c|c|c|c|c|}
\hline Ref & Method & $\begin{array}{c}\text { Learning } \\
\text { Technique }\end{array}$ & $\begin{array}{c}\text { Handle } \\
\text { Data } \\
\text { Fading }\end{array}$ & $\begin{array}{c}\text { Input } \\
\text { Data } \\
\text { Form }\end{array}$ & Parameters Required & Data Structure & $\begin{array}{l}\text { Window } \\
\text { Models }\end{array}$ & $\begin{array}{l}\text { Outlier } \\
\text { Detec. }\end{array}$ & $\begin{array}{c}\text { High } \\
\text { Dimen } \\
\text { Data }\end{array}$ \\
\hline [30] & $\mathrm{BIRCH}$ & Two-Phase & $\mathrm{NO}$ & Example & Branching Factor, Threshold & Feature Vector & Landmark & YES & YES \\
\hline [23] & CluStream & Two-Phase & NO & Example & Number of Clusters, Time Window & Feature Vector & Tilted-Time & $\begin{array}{c}\text { Statistical- } \\
\text { Based }\end{array}$ & NO \\
\hline$[32]$ & HPstream & Two-Phase & YES & Example & $\begin{array}{l}\text { Max. Number of Clusters, Average Number of Projection } \\
\text { Dimension }\end{array}$ & Prototype Array & Slid-window & NO & YES \\
\hline$[33]$ & Ducstream & Two-Phase & NO & Example & Threshold of Density of Grid Cells & Prototype Array & Slid-window & $\begin{array}{l}\text { Density- } \\
\text { Based }\end{array}$ & NO \\
\hline [34] & DenStream & Two-Phase & YES & Example & Cluster Radius Threshold, Data Fading Rate & Feature Vector & Fading & $\begin{array}{l}\text { Density- } \\
\text { Based }\end{array}$ & NO \\
\hline [35] & UMicro & Two-Phase & NO & Example & Number of Clusters, Number of Micro-clusters, Timestamp & Feature Vector & Slid-window & $\mathrm{NO}$ & YES \\
\hline [36] & SDStream & Two-Phase & YES & Example & Eps, Mean and Max. Number of Micro-clusters & Feature Vector & Slid-window & $\begin{array}{l}\text { Density- } \\
\text { Based }\end{array}$ & NO \\
\hline [37] & $\begin{array}{l}\text { Wavelet- } \\
\text { synopsis }\end{array}$ & Two-Phase & NO & Variable & Number of Clusters, Threshold of Cluster Boundary & Feature Vector & Slid-window & NO & YES \\
\hline [39] & SPE-Cluster & Incremental & $\mathrm{NO}$ & Variable & Size of Sliding \& Basic Window & Prototype Array & Tilted-Time & $\mathrm{NO}$ & $\mathrm{NO}$ \\
\hline [40] & FlockStream & Incremental & YES & Example & $\begin{array}{l}\text { Max. Number of Iteration, Visible Range, Velocity Vector } \\
\text { of agent }\end{array}$ & Feature Vector & Fading & $\begin{array}{l}\text { Density- } \\
\text { Based }\end{array}$ & NO \\
\hline [41] & EXCC & Two-Phase & YES & Example & Eps, Max. Number of Neighbours, Grid Size & Data Grid & Landmark & $\begin{array}{l}\text { Density- } \\
\text { Based }\end{array}$ & YES \\
\hline [42] & $\begin{array}{c}\text { HASTREA } \\
\mathrm{M} \\
\end{array}$ & Incremental & YES & Example & $\begin{array}{l}\text { Eps, Min. Number of Data Points, Min. Spanning Tree, } \\
\text { Max. Size of Micro-cluster }\end{array}$ & Feature Vector & Fading & $\begin{array}{l}\text { Density- } \\
\text { Based }\end{array}$ & YES \\
\hline$[1]$ & SoStream & Incremental & YES & Example & $\begin{array}{l}\text { Minimum Distance, Threshold of Closest Neighbour, } \\
\text { Threshold of Overlapped }\end{array}$ & Feature Vector & Fading & $\begin{array}{l}\text { Density- } \\
\text { Based }\end{array}$ & NO \\
\hline [43] & CEDAS & Incremental & YES & Variable & $\begin{array}{l}\text { Eps, MinPts, Max. Number of Neighbours, Max. Number of } \\
\text { micro-clusters }\end{array}$ & Data Grid & Landmark & $\begin{array}{c}\text { Density- } \\
\text { Based }\end{array}$ & NO \\
\hline
\end{tabular}




\section{Data Stream Mining Tools}

Tools of programming in information mining which developed rapidly in course of most recent twenty years. The market headers incorporate WEKA, MATLAB, Oracle DM, IBM DW, TANAGRA, Microsoft SQL Server DM RapidMiner, and .NET system.

A significant number of those frameworks function admirably of static data content of generally little volumes; however they don't explicitly intended of taking care of data streams and especially huge data streams. As of late, business frameworks for streaming data mining began in arrive, as instance, VFML, SAMOA, MOA and. MOA structure is an open-source benchmarking programming for data streams that is based on work by WEKA. It has a group of stream methods and an assortment of assessment measures. MOA has considered stream order methods; nonetheless, as of late they include stream data grouping assessment device. They permit creating and executing tests of mining information as well as AI strategies on developing streaming data. They incorporates an assortment in students and generating streams which may used from the introducing UI. SAMOA is an instrument of mining huge streaming data. Objective in SAMOA may give structure to manage stream mining data utilizing cloud computation. They gives group of circulated streaming methods of open AI and information mining strategies composed characterization, grouping, regression, just programming devices to grow new methodologies and methods. It can run on many dispersed stream handling stages like Apache Storm and Samza. At last, VFML (quick AI) is C-based programming bundle for mining fast data streams and extremely enormous datasets. It is comprised of three fundamental substances. The principal part is a group of apparatuses and APIs that assist a developer with creating information mining algorithms. The $2^{\text {nd }}$ comprise is group from executions average information mining methods. The $3^{\text {rd }}$ substance is group in versatile information mining methods which developed by Pedro Domingos and his followers. VFML include an assortment from instruments in developing data processing: cleaning, inspecting, and parting those to queue of group test [44].

\section{Conclusion}

Streaming data are infinitive, huge and advance over the long run which makes mining an exceptionally difficult undertaking. In this research, a survey of essential information and specialized foundations pertinent to the examination completed in this research was given. The initial segment of this research builds up a fundamental comprehension Big Data rules which underline of velocity attributes in Big Data as stream data. In this case the grounds that the research is worried about data stream mining, tending to velocity as a specific part of Big Data investigation. The requirement for modern data stream methods is featured in the subsequent part. The examination additionally addresses a significant issue with respect to how results can be assessed. The assessment estimates that have been referenced may be utilized to survey the exhibition of data stream mining methods. The last piece of this research creates the establishment of data stream mining with the accentuation on model development and concept drift as the main viewpoints to be utilized in data stream mining methods. The exploration momentarily investigated the two existing computational methodologies, for example incremental and two-stage learning, for data stream mining. The current data stream methods are deficient with regards to general clear strides for dissecting new approaching information chunks. In any case, most of existing data stream arrangements is adjusting the strategies for static data to work with data stream setting. The research proposed presenting strategies which make the accompanying: led prevalent, better performance outputs, produces outputs about effective way, control anomalies and adaptation.

\section{References}

[1] C. Isaksson, "New Outlier Detection Techniques For Data Streams," Thesis, Southern Methodist University, Bobby B. Lyle School of Engineering, 2016.

[2] A. K. Jain, "Data clustering : 50 years beyond K-means," Pattern Recognit. Lett. , J. Sci. 
ELSVIER, vol. 31, no. 8, pp. 651-666, 2010.

[3] A. Al Abd Alazeez, S. Jassim, and H. Du, "EINCKM: An Enhanced Prototype-based Method for Clustering Evolving Data Streams in Big Data," Proc. 6th Int. Conf. Pattern Recognit. Appl. Methods, no. Icpram, pp. 173-183, 2017.

[4] M. Hassani, "Efficient Clustering of Big Data Streams," Thesis, Eindhven University of Technology, Depatment of Computer Science, 2015.

[5] A. Bifet, A. Carvalho, and J. Gama, "BigData Stream Mining," Lecture Note- Telecom ParisTech, 75634 Paris Cedex 13, FRANCE, 2017.

[6] D. A. Marcos, N. C. Rodrigo, B. Silvia, A. S. N. Marco, and B. Rajkumar, "Big Data Computing and Clouds: Trends and Future Directions," J. Parallel Distrib. Comput., no. arXiv:1312.4722v2, pp. 1-44, 2014.

[7] I. A. T. Hashem, I. Yaqoob, N. Badrul Anuar, S. Mokhtar, A. Gani, and S. Ullah Khan, "The rise of 'Big Data' on cloud computing: Review and open research issues," Inf. Syst., vol. 47, pp. 98-115, Aug. 2014.

[8] Y. B. Wah and I. R. Ibrahim, "Using data mining predictive models to classify credit card applicants," 2010 6th Int. Conf. Adv. Inf. Manag. Serv., pp. 394-398, 2010.

[9] M. Rose, "TechTarget," TechTarget, 2015. [Online]. Available: http://searchbusinessanalytics.techtarget.com/definition/association-rules-in-data-mining.

[10] S. Lv and H. Kim, "applied sciences A Review of Data Mining with Big Data towards Its Applications in the Electronics Industry," no. Dm, pp. 1-34, 2018.

[11] H. L. Nguyen, Y. K. Woon, and W. K. Ng, "A survey on data stream clustering and classification," Knowl. Inf. Syst. Springer, pp. 535-569, 2015.

[12] P. Chauhan, "A Review on Outlier Detection Techniques on Data Stream by Using Different Approaches of K-Means Algorithm," Int. Conf. Adv. Comput. Eng. Appl. IMS Eng. Coll. Ghaziabad, India, pp. 580-585, 2015.

[13] A. T. Y. T. A. A. Alazeez, "HPPD: A Hybrid Parallel Framework of Partition-based and Density-based Clustering Algorithms in Data Streams Ammar," Raf. J. Comp. Math's., vol. 1, no. 1, pp. 67-82, 2020.

[14] M. Rahmani and G. K. Atia, "Robust PCA with Concurrent Column and Element-wise Outliers," Fifty-Fifth Annu. Allert. Conf. Allert. House, UIUC, Illinois, USA Oct. 3-6, 2017, IEEE, pp. 332-337, 2017.

[15] A. T. Y. Al Abd Alazeez, S. Jassim, and H. Du, "SLDPC : Towards Second Order Learning for Detecting Persistent Clusters in Data Streams," 2018 10th Comput. Sci. Electron. Eng., vol. 978-1-5386, pp. 248-253, 2018.

[16] M. Oliveira and J. Gama, "A Framework to Monitor Clusters' Evolution Applied to Economy and Finance Problems," Intell. Data Anal. 16, 1, 93-111, 2012.

[17] L. Zheng, H. Huo, Y. Guo, and T. Fang, "Supervised Adaptive Incremental Clustering for data stream of chunks," Neurocomputing, vol. 219, no. September 2016, pp. 502-517, 2017.

[18] A. T. Y. A. A. A. Al Abd Alazeez, "AEPRD: An Enhanced Algorithm for Predicting Results of Orthodontic Operations," J. Educ. Sci., vol. 30, no. 1, pp. 173-190, 2021.

[19] T. S. Sethi and M. Kantardzic, "On the reliable detection of concept drift from streaming unlabeled data," Expert Syst. Appl., vol. 82, pp. 77-99, 2017.

[20] A. T. Y. T. A. A. Alazeez, "DED: Drift Principle in Educational Evolved Data," Tikrit J. Pure Sci., vol. 26, no. 2, pp. 118-125, 2021.

[21] S. Saha and S. Bandyopadhyay, "A New Measure of Stability of Clustering Solutions: Application to Data Partitioning," 2009 Int. Conf. Adapt. Intell. Syst., 2009.

[22] A. Al Abd Alazeez, S. Jassim, and H. Du, "EDDS: An Enhanced Density-Based Method for Clustering Data Streams," 2017 46th Int. Conf. Parallel Process. Work., pp. 103-112, 2017.

[23] C. Aggarwal, J. Han, J. Wang, and P. Yu, "A Framework for Clustering Evolving Data Streams," Proc. 29th VLDB Conf. Ger., 2003. 
[24] A. Amini, “An Adaptive Density-Based Method for Clustering Evolving Data Streams," Thesis, University of Malaya Kuala Lumpur, Department of Computer Science and Information Technology, 2014.

[25] M. Mousavi, A. A. Bakar, and M. Vakilian, "Data stream clustering algorithms: A review," Int. J. Adv. Soft Comput. its Appl., vol. 7, no. Specialissue3, pp. 1-15, 2015.

[26] J. Silva, E. Faria, R. Barros, E. Hruschka, and A. Carvalho, "Data Stream Clustering : A Survey," ACM Comput. Surv., pp. 1-37, 2013.

[27] A. Al Abd Alazeez, S. Jassim, and H. Du, "TPICDS: A Two-phase Parallel Approach for Incremental Clustering of Data Streams," 24th Int. Eur. Conf. Parallel Distrib. Comput., 2018.

[28] M. Carnein, D. Assenmacher, and H. Trautmann, "An Empirical Comparison of Stream Clustering Algorithms," Proc. Comput. Front. Conf. ZZZ - CF'17, pp. 361-366, 2017.

[29] B. Aaron, D. E. Tamir, N. D. Rishe, and A. Kandel, "Dynamic Incremental K-means Clustering,” 2014 Int. Conf. Comput. Sci. Comput. Intell. Dyn., pp. 308-313, 2014.

[30] T. Zhang, R. Ramakrishnan, and M. Livny, "BIRCH: An Efficient Data Clustering Databases Method for Very Large Databases," ACM, SIGMOD, Int. Conf. Manag. Data, vol. 1, pp. 103114, 1996.

[31] S. Guha, N. Mishra, R. Motwani, and L. O'Callaghan, "Clustering Data Streams," 0-76950850-2/00\$10.00 02000 IEEE, pp. 359-366, 2000.

[32] C. C. Aggarwal, J. Han, J. Wang, and P. S. Yu, "A Framework for Projected Clustering of High Dimensional Data Streams," Proc. Thirtieth Int. Conf. Very large data bases, vol. 30, p. 863, 2004.

[33] J. Gao, J. Li, Z. Zhang, and P. N. Tan, "An incremental data stream clustering algorithm based on dense units detection," Adv. Knowl. Discov. Data Min., pp. 420-425, 2005.

[34] F. Cao, M. Ester, W. Qian, and A. Zhou, "Density-based clustering over an evolving data stream with noise," Proc. Sixth SIAM Int. Conf. Data Min., vol. 2006, pp. 328-339, 2006.

[35] C. C. Aggarwal and P. S. Yu, "A Framework for Clustering Uncertain Data Streams," IEEE 24th Int. Conf. Data Eng. (ICDE 2008), pp. 150-159, 2008.

[36] J. Ren and R. Ma, "Density-based data streams clustering over sliding windows," 6th Int. Conf. Fuzzy Syst. Knowl. Discov. FSKD 2009, vol. 5, pp. 248-252, 2009.

[37] H.-H. CHEN, B.-L. SHI, J.-B. QIAN, and Y.-F. CHEN, "Wavelet Synopsis Based Clustering of Parallel Data Streams," J. Softw., vol. 21, no. 4, pp. 644-658, 2010.

[38] P. Kranen, I. Assent, C. Baldauf, and T. Seidl, "The ClusTree : indexing micro-clusters for anytime stream mining," pp. 249-272, 2011.

[39] C. Ling, Z. L. Jun, and T. Li, "A clustering algorithm for multiple data streams based on spectral component similarity,” ELSVIER, J. Inf. Sci., vol. 183, no. 1, pp. 35-47, 2012.

[40] A. Forestiero, C. Pizzuti, and G. Spezzano, "A single pass algorithm for clustering evolving data streams based on swarm intelligence," Data Min. Knowl. Discov., vol. 26, no. 1, pp. 126, 2013.

[41] V. Bhatnagar, S. Kaur, and S. Chakravarthy, "Clustering data streams using grid-based synopsis," Knowl. Inf. Syst., vol. 41, no. 1, pp. 127-152, 2014.

[42] P. Spaus, A. Cuzzocrea, and T. Seidl, "Adaptive Stream Clustering Using Incremental Graph Maintenance," pp. 49-64, 2015.

[43] R. Hyde, P. Angelov, and A. R. MacKenzie, "Fully online clustering of evolving data streams into arbitrarily shaped clusters," Inf. Sci. (Ny)., vol. 382-383, pp. 96-114, 2017.

[44] T. Rajesh and K. V. . Rao, "Hybrid Clustering Algorithm for Time Series Data Stream: Current State of the Art," Int. J. Innov. Res. Comput. Commun. Eng., vol. 5, no. 3, pp. 57865794, 2017. 